\title{
Efficacy, safety and prognostic factors in patients with refractory metastatic colorectal cancer treated with trifluridine/tipiracil plus bevacizumab in a real- world setting
}

Nieves Martínez-Lago ( $\square$ Nieves.Purificacion.Martinez.Lago@sergas.es )

University Hospital A Coruña, A Coruña

Teresa Calleja Chucla

University Hospital A Coruña, A Coruña

Beatriz Alonso de Castro

University Hospital A Coruña, A Coruña

Rafael Varela Ponte

University Hospital Santiago de Compostela

Cristina Reboredo Rendo

University Hospital A Coruña, A Coruña

Martin Igor Gomez-Randulfe Rodriguez

University Hospital A Coruña, A Coruña

Sofia Silva Diaz

University Hospital A Coruña, A Coruña

Begoña Graña Suarez

University Hospital A Coruña, A Coruña

Juan de la Camara Gomez

University Hospital A Coruña, A Coruña

Fernando Busto López

University Hospital A Coruña, A Coruña

María Mateos Salvador

University Hospital A Coruña, A Coruña

Margarita Reboredo Lopez

University Hospital A Coruña, A Coruña

\section{Research Article}

Keywords: Bevacizumab, metastatic colorectal cancer, trifluridine/tipiracil

Posted Date: January 14th, 2022 
DOI: https://doi.org/10.21203/rs.3.rs-1251622/v1

License: (c) (1) This work is licensed under a Creative Commons Attribution 4.0 International License. Read Full License 


\section{Abstract}

We evaluated the efficacy and safety of trifluridine/tipiracil (TAS-102) plus bevacizumab in treating refractory metastatic colorectal cancer $(\mathrm{mCRC})$ in a retrospective, observational study. Patients refractory or intolerant to standard therapies received TAS-102 (30-35 mg/ $\mathrm{m}^{2}$ twice daily on days $1-5$ and days 812 every 28 days) plus bevacizumab $5 \mathrm{mg} / \mathrm{kg}$ on days 1 and 15 . Clinical and pathological characteristics, overall response rate (ORR) and disease control rate (DCR), overall survival (OS) and progression-free survival (PFS) data were collected and analysed. Thirty-five patients were treated from July 2019 to October 2021 (median age 64 years). The majority of patients (68.6\%) were receiving TAS-102 plus bevacizumab as third-line treatment. Patients received a median of 4 (range 2-15) cycles of treatment. Among 31 patients evaluable for response (88.6\%), ORR and DCR were $3.2 \%$ and $51.6 \%$, respectively. After a median 11.6 months' follow-up, median PFS was 4.3 (95\% confidence interval [Cl] 3.4-5.1) months and median OS was $9.3(95 \% \mathrm{Cl} 6.6-12.1)$ months. The most common grade $3-4$ toxicities were neutropenia, asthenia and nausea/vomiting, and there were no treatment-related deaths. This real-world study confirms the efficacy and safety of TAS-102 plus bevacizumab in patients with refractory mCRC.

\section{Introduction}

Colorectal cancer (CRC) is the second highest cause of cancer-related death worldwide, with an estimated 1.8 million new cases and $>880,000$ deaths in $2018^{[1]}$. Evidence-based guidelines recommend cytotoxic chemotherapy (e.g. oxaliplatin, irinotecan or fluoropyrimidines) as first- and second-line treatment in patients with metastatic CRC (mCRC), with the addition of anti-epidermal growth factor receptor (EGFR) agents (i.e. cetuximab or panitumumab) in those with wild-type RAS tumours or the anti-vascular endothelial growth factor (VEGF) agent bevacizumab ${ }^{[2]}$. However, a high proportion of patients develop progressive disease (PD) after receiving standard chemotherapy, with $>40 \%$ receiving at least three lines

of treatment ${ }^{[3]}$. Treatment recommendations now include the multi-kinase inhibitor regorafenib and trifluridine/tipiracil (TAS-102) as third-line treatment options in these patients ${ }^{[2]}$.

The efficacy and safety of TAS-102, a combination of a thymidine-based nucleic acid analogue (trifluridine) and a thymidine phosphorylase inhibitor (tipiracil), have been demonstrated in clinical studies of patients with previously treated $\mathrm{mCRC}$, with significantly improved overall survival (OS) compared with placebo ${ }^{[4-6]}$. An exploratory analysis of the RECOURSE clinical study ${ }^{[4]}$ identified patients with low tumour burden and less aggressive disease (i.e. $\geq 18$ months since metastatic disease diagnosis) as having improved survival outcomes with TAS-102 monotherapy ${ }^{[7]}$.

The survival benefits with TAS-102 monotherapy are modest and there is a need for improved treatment options in patients with refractory MCRC. Several phase I/II and phase II studies have investigated the efficacy and safety of TAS-102 in combination with bevacizumab ${ }^{[8-13]}$. These studies included the singlearm Japanese C-TASKFORCE study in 25 patients with refractory $\mathrm{mCRC}$, which reported a centrallyassessed median progression-free survival (PFS) of 3.7 months and a median OS of 11.4 months with 
TAS-102 plus bevacizumab ${ }^{[9]}$. In a Danish randomised study in 93 patients with refractory mCRC, TAS102 plus bevacizumab was associated with significantly improved median PFS (4.6 vs 2.6 months, hazard ratio $[\mathrm{HR}] 0.45, \mathrm{p}=0.001)$ and median OS (9.4 vs 6.7 months, $\mathrm{HR}, 0.55, \mathrm{p}=0.028$ ) compared with TAS-102 monotherapy ${ }^{[10]}$.

Previous retrospective studies of Japanese patients with refractory mCRC have also indicated that the TAS-102 plus bevacizumab combination provides significant survival benefits compared with TAS-102 monotherapy in routine clinical practice ${ }^{[14-16]}$; however, real-world data on the use of TAS-102 plus bevacizumab in non-Asian populations are limited. The aim of this real-world study was to evaluate the efficacy, safety and prognostic factors of TAS-102 plus bevacizumab in patients with refractory $\mathrm{mCRC}$ in routine clinical practice in Spain.

\section{Results}

\section{Population characteristics}

Thirty-five patients were treated with TAS-102 plus bevacizumab between July 2019 and October 2021 and were included in this study. Patient characteristics are summarised in Table 1. Patients had a median (range) age of $65(41-82)$ years and $31.4 \%$ were aged $\geq 70$ years. The majority of patients $(88.6 \%)$ had undergone primary tumour resection, $77.1 \%$ had an Eastern Cooperative Oncology performance status (ECOG PS) of $0-1,80.0 \%$ had liver metastases, and $71.4 \%$ were diagnosed with metastatic disease $\geq 18$ months before starting TAS-102 plus bevacizumab. Previous treatment included anti-VEGF therapy in $94.3 \%$ of patients; $68.6 \%$ were receiving TAS- 102 plus bevacizumab as third-line treatment. None of the patients had previously received regorafenib. 
Table 1

Study population characteristics.

\section{Characteristics}

Age, years

Median (range)

$>70$ years, $\mathrm{n}(\%)$

Gender, n (\%)

Male

Female

ECOG PS, n (\%)

$0-1$

2

Tumour location, $\mathrm{n}(\%)$

Right-sided

Left-sided

Rectum

Histological grade, $\mathrm{n}(\%)$

Low grade (G1-G2)

High grade (G3)

Unknown

$R A S / B R A F$ mutational status, $\mathrm{n}(\%)$

$R A S / B R A F$ wild type

RAS mutated

$B R A F$ mutated

Mismatch repair protein expression, $\mathrm{n}(\%)$

Conserved

ECOG PS Eastern Cooperative Oncology Group performance status, G grade, No. number, TAS-102 trifluridine/tipiracil, VEGF vascular endothelial growth factor.

TAS-102 was started at a reduced dose $\left(30 \mathrm{mg} / \mathrm{m}^{2}\right)$ in seven patients $(20.0 \%)$ and no patients started bevacizumab at reduced doses. Prophylactic granulocyte colony-stimulating factor (G-CSF) treatment was administered to five patients (14.3\%). Patients received a median of 4 cycles of TAS-102 plus

bevacizumab (range $2-15$ cycles).
$\mathrm{N}=35$

$65(41-82)$

11 (31.4)

22 (62.9)

13 (37.1)

27 (77.1)

8 (22.9)

$4(11.4)$

17 (48.6)

$14(40.0)$

$22(62.9)$

2 (5.7)

11 (31.4)

16 (45.7)

17 (48.6)

2 (5.7)

35 (100) 


\section{Characteristics}

$\mathbf{N}=35$

Tumour presentation, $\mathrm{n}$ (\%)

Synchronous

23 65.7)

Metachronous

Primary tumour surgery, $\mathrm{n}(\%)$

$31(88.6)$

Previous anti-VEGF therapy, $\mathrm{n}$ (\%)

$33(94.3)$

Line of TAS-102 + bevacizumab treatment, $n(\%)$

3

4

$\geq 5$

$7(20.0)$

Liver metastases, $\mathrm{n}(\%)$

$28(80.0)$

No. of metastatic locations, $n(\%)$

$<3$

$\geq 3$

Time from metastatic disease diagnosis, $\mathrm{n}(\%)$

$<18$ months

$\geq 18$ months

Tabernero prognostic classification, $\mathrm{n}(\%)$

Best

Good

$12(34.3)$

Poor $20(57.1)$

ECOG PSEastern Cooperative Oncology Group performance status, G grade, No. number, TAS-102 trifluridine/tipiracil, VEGF vascular endothelial growth factor.

TAS-102 was started at a reduced dose $\left(30 \mathrm{mg} / \mathrm{m}^{2}\right)$ in seven patients $(20.0 \%)$ and no patients started bevacizumab at reduced doses. Prophylactic granulocyte colony-stimulating factor (G-CSF) treatment was administered to five patients (14.3\%). Patients received a median of 4 cycles of TAS-102 plus bevacizumab (range $2-15$ cycles).

\section{Efficacy}

In total, 31 of 35 patients (88.6\%) were evaluable for response; two patients $(5.7 \%)$ were not evaluable due to an early death, and two patients $(5.7 \%)$ had response assessment pending at the time of the analysis. After a median follow-up of 10.8 months, 15 patients $(48.4 \%)$ had PD, one (3.2\%) had achieved 
partial response (PR) and no patients had complete response (CR) (Table 2). The overall response rate (ORR) and disease control rate (DCR) were $3.2 \%$ and $51.6 \%$, respectively.

Table 2

Response rate.

\begin{tabular}{|l|l|}
\hline Response, $\mathbf{n}(\%)$ & $\mathbf{N}=\mathbf{3 1}$ \\
\hline Best overall response & \\
\hline CR & 0 \\
\hline PR & $1(3.2)$ \\
\hline SD & $15(48.4)$ \\
\hline PD & $15(48.4)$ \\
\hline ORR (CR + PR) & $1(3.2)$ \\
\hline DCR (CR + PR + SD) & $16(51.6)$ \\
\hline$C R$ complete response, $D C R$ disease control rate, ORR overall response rate, $P D$ progressive disease, \\
\hline$P R$ partial response, $S D$ stable disease. \\
\hline
\end{tabular}

Based on Kaplan-Meier estimates, patients had a median PFS of 4.3 months (95\% confidence interval [CI] 3.4-5.1 months) (Figure 1a) and a median OS of 9.3 months (95\% $\mathrm{Cl} 6.6-12.1$ months) (Figure 1b).

In the univariate regression analysis, prognostic factors associated with significantly improved OS were the absence (vs presence) of peritoneal metastases and grade 1-2 (vs grade 3 ) tumour histological grade (Table 3). The absence of peritoneal metastases was also associated with significantly improved PFS, whereas $<3$ ( $v s \geq 3$ ) metastatic sites was associated with significantly worse PFS. 
Table 3

Univariate analysis of prognostic factors for progression-free and overall survival.

\begin{tabular}{|c|c|c|c|c|c|c|}
\hline Characteristic & $\begin{array}{l}\text { PFS, } \\
\text { months }\end{array}$ & $\mathrm{HR}(95 \% \mathrm{Cl})^{\mathrm{a}}$ & $\begin{array}{l}\text { p- } \\
\text { value }^{a}\end{array}$ & $\begin{array}{l}\text { OS, } \\
\text { months }\end{array}$ & $\mathrm{HR}(95 \% \mathrm{Cl})^{\mathrm{a}}$ & $\begin{array}{l}\text { p- } \\
\text { value }\end{array}$ \\
\hline \multicolumn{7}{|l|}{ Gender } \\
\hline Male & 4.2 & $\begin{array}{l}1.233(0.6- \\
2.7)\end{array}$ & 0.599 & 8.1 & $\begin{array}{l}1.186(0.5- \\
2.9)\end{array}$ & 0.704 \\
\hline Female & 4.7 & - & - & 11.1 & - & - \\
\hline \multicolumn{7}{|l|}{ ECOG PS } \\
\hline $0-1$ & 4.7 & $\begin{array}{l}0.652(0.3- \\
1.6)\end{array}$ & 0.336 & 9.3 & $\begin{array}{l}0.517(0.2- \\
1.4)\end{array}$ & 0.182 \\
\hline 2 & 3.5 & - & - & 8.1 & - & - \\
\hline \multicolumn{7}{|c|}{ Histological grade } \\
\hline G1-G2 & 4.7 & $\begin{array}{l}0.424(0.1- \\
1.9)\end{array}$ & 0.247 & 10.6 & $\begin{array}{l}0.087(0.1- \\
0.5)\end{array}$ & 0.001 \\
\hline G3 & 2.5 & - & - & 3.4 & - & - \\
\hline \multicolumn{7}{|c|}{ Tumour presentation } \\
\hline Synchronous & 4.0 & $\begin{array}{l}2.391(0.9- \\
5.7)\end{array}$ & 0.053 & 9.3 & $\begin{array}{l}1.017(0.4- \\
2.6)\end{array}$ & 0.971 \\
\hline Metachronous & 6.0 & - & - & 10.6 & - & - \\
\hline \multicolumn{7}{|l|}{ Tumour surgery } \\
\hline No & 2.8 & $\begin{array}{l}1.044(0.4- \\
3.0)\end{array}$ & 0.937 & 5.3 & $\begin{array}{l}1.396(0.5- \\
4.3)\end{array}$ & 0.562 \\
\hline Yes & 4.3 & - & - & 9.3 & - & - \\
\hline \multicolumn{7}{|l|}{ Metastatic sites } \\
\hline$<3$ & 3.0 & $\begin{array}{l}2.286(1.0- \\
5.1)\end{array}$ & 0.001 & 5.2 & $\begin{array}{l}2.790(0.9- \\
7.8)\end{array}$ & 0.06 \\
\hline$\geq 3$ & 8.2 & - & - & 17.7 & - & - \\
\hline \multicolumn{7}{|c|}{ Liver metastases } \\
\hline No & 17.5 & $\begin{array}{l}0.309(0.1- \\
1.1)\end{array}$ & 0.069 & 8.1 & $\begin{array}{l}0.782(0.2- \\
2.7)\end{array}$ & 0.696 \\
\hline
\end{tabular}

Cl confidence interval, ECOG PS Eastern Cooperative Oncology Group performance status, $G$ grade, $H R$ hazard ratio, NR not reached, OS overall survival, PFS progression-free survival.

asignificant values are indicated in bold text. 


\begin{tabular}{|c|c|c|c|c|c|c|}
\hline Characteristic & $\begin{array}{l}\text { PFS, } \\
\text { months }\end{array}$ & $\mathrm{HR}(95 \% \mathrm{Cl})^{\mathrm{a}}$ & $\begin{array}{l}\text { p- } \\
\text { value }^{a}\end{array}$ & $\begin{array}{l}\text { OS, } \\
\text { months }\end{array}$ & $\mathrm{HR}(95 \% \mathrm{Cl})^{\mathrm{a}}$ & $\begin{array}{l}\text { p- } \\
\text { value }\end{array}$ \\
\hline Yes & 4.0 & - & - & 9.3 & - & - \\
\hline \multicolumn{7}{|c|}{ Peritoneal metastases } \\
\hline No & 4.7 & $\begin{array}{l}0.393(0.1- \\
0.9)\end{array}$ & 0.046 & 9.3 & $\begin{array}{l}0.437(0.2- \\
1.0)\end{array}$ & 0.07 \\
\hline Yes & 2.8 & - & - & 5.6 & - & - \\
\hline \multicolumn{7}{|c|}{ Time from metastatic disease diagnosis } \\
\hline$\geq 18$ months & 4.3 & $\begin{array}{l}0.763(0.3- \\
1.8)\end{array}$ & 0.523 & 9.3 & $\begin{array}{l}0.871(0.3- \\
2.3)\end{array}$ & 0.780 \\
\hline$<18$ months & 4.8 & - & - & 17.7 & - & - \\
\hline \multicolumn{7}{|c|}{ Tabernero prognostic classification } \\
\hline Best & NR & - & 0.069 & NR & - & 0.071 \\
\hline Good & 5.0 & - & - & 11.1 & - & - \\
\hline Poor & 3.5 & - & - & 7.9 & - & - \\
\hline \multicolumn{7}{|c|}{$\begin{array}{l}\text { C/ confidence interval, ECOG PS Eastern Cooperative Oncology Group performance status, G grade, } \\
H R \text { hazard ratio, NR not reached, OS overall survival, PFS progression-free survival. }\end{array}$} \\
\hline
\end{tabular}

\section{Safety}

The most common adverse events (AEs) of any grade were neutropenia (74.3\%), asthenia $(65.7 \%)$, anaemia (54.8\%) and thrombocytopenia (34.3\%) (Table 4). The most frequent grade 3-4 AEs were neutropenia (45.7\%), asthenia (17.1\%) and nausea/vomiting (8.6\%). There were no reports of febrile neutropenia and no treatment-related deaths. Neutropenia was managed by reducing the dose of TAS102 in five patients (38.1\%) and administration of G-CSF prophylaxis in five patients (33.3\%), while in the other patients, treatment was delayed until recovery. No patients required a dose reduction of bevacizumab. 
Table 4

Summary of the most frequent all grade and grade $3 / 4$ adverse events.

\begin{tabular}{|lll|}
\hline AE, $\mathrm{n}(\%)$ & All Grades & Grade 3-4 \\
\hline Neutropenia & $26(74.3)$ & $16(45.7)$ \\
\hline Asthenia & $23(65.7)$ & $6(17.1)$ \\
\hline Anaemia & $17(54.8)$ & $2(5.7)$ \\
\hline Thrombocytopenia & $12(34.3)$ & $2(5.7)$ \\
\hline Diarrhoea & $12(34.3)$ & $2(5.7)$ \\
\hline Hepatic function abnormalities & $8(22.9)$ & 0 \\
\hline Nausea/vomiting & $6(17.1)$ & $3(8.6)$ \\
\hline Bleeding & $4(11.4)$ & 0 \\
\hline Hypertension & $2(5.7)$ & $1(2.9)$ \\
\hline Venous thromboembolism & 0 & 0 \\
\hline Febrile neutropenia & 0 & 0 \\
\hline AE adverse event. & & \\
\hline
\end{tabular}

\section{Discussion}

In this real-world study of TAS-102 plus bevacizumab treatment in patients with refractory mCRC, efficacy and safety data were generally consistent with those of previous clinical studies, including the Japanese C-TASKFORCE study ${ }^{[9]}$ and the Danish phase II study ${ }^{[10]}$. The ORR in our study (3.2\%) was slightly higher than that reported in C-TASKFORCE ( $0 \%$ by central assessment $)^{[9]}$ and the Danish study $(2 \%)^{[10]}$, whereas the DCR was slightly lower in our study $(51.6 \%)$ than in earlier studies $(64 \% \text { and } 67 \% \text {, respectively })^{[9,10]}$. In our study, the median PFS (4.3 months) was similar to that reported in the earlier studies (3.7 and 4.6 months, respectively), while the median OS (9.3 months) was similar to that of the Danish study ( 9.4 months $)^{[10]}$, but slightly lower than in C-TASKFORCE (11.4 months) ${ }^{[9]}$.

The efficacy of TAS-102 plus bevacizumab in our study was also generally comparable to that reported in previous real-world retrospective studies of Japanese patients with refractory $\mathrm{mCRC}$, in which the median PFS with TAS-102 plus bevacizumab was 3.7 months ${ }^{[16]}$ or 4.4 months $^{[14]}$, and the median OS ranged from 8.6 to 14.4 months $^{[14-16]}$.

Patients with refractory $\mathrm{mCRC}$ often have poor prognosis ${ }^{[17]}$. In our real-world study, $22.9 \%$ of patients had an ECOG PS of 2 and therefore may be more representative of patients with refractory mCRC in routine clinical practice than the previous C-TASKFORCE and Danish clinical studies, which excluded 
patients with ECOG PS of $2^{[9,10]}$. In the previous Japanese real-world studies of TAS-102 plus bevacizumab, the proportion of patients with ECOG PS of 2 (or modified Glasgow prognostic score of 2) was also much lower $(1.4-4.8 \%)^{[14-16]}$ than in our study. Therefore, our study indicates that TAS-102 plus bevacizumab continues to be effective in patients with refractory $\mathrm{mCRC}$ and poor performance status scores.

In the univariate analysis of prognostic factors for survival, our study showed that OS and PFS were significantly improved in patients without peritoneal metastases, and those with low tumour histological grade had significantly improved OS. However, patients with low tumour burden ( 3 metastatic sites) had significantly worse PFS compared with those with $\geq 3$ metastatic sites. Although this result seems counterintuitive, the low tumour burden may be an indicator of treatment intensity or the finding may be a statistical artefact associated with the small population size of our study. Another study found no difference in survival outcomes between patients CRC with three versus four metastatic sites ${ }^{[18]}$. Moreover, a large database analysis of the correlates of survival showed that the organ affected by metastasis was an important determinant of survival ${ }^{[19]}$. Further research is needed to determine whether it is the number of metastatic sites or the organs affected by metastases that has the greatest impact on survival outcomes.

Previous studies have identified other baseline prognostic factors associated with improved clinical outcomes with TAS-102 (either as monotherapy or combined with bevacizumab), including modified Glasgow prognostic score ${ }^{[20]}$, the Tabernero prognostic factors (i.e. low tumour burden, less aggressive disease [ $\geq 18$ months since diagnosis of metastatic disease] and liver metastases) ${ }^{[7]}$, high lymphocyteto-monocyte ratio $(\geq 3.18)^{[21]}$, and the TAS-RECOSMO predictive model (i.e. general status, neutrophil-tolymphocyte ratio, KRAS, NRAS and BRAF mutation status, carcinoembryonic antigen [CEA] and alkaline phosphatase [ALP] levels, and time since metastatic disease diagnosis) ${ }^{[17]}$. However, our study did not identify liver metastases or the time since diagnosis of metastasis $<18$ months (i.e. the Tabernero factors) as being prognostic of OS or PFS, and we did not examine mutational status, CEA or ALP levels, or lymphocyte-to-monocyte or neutrophil-to-lymphocyte ratios as potential prognostic factors.

In our study, TAS-102 plus bevacizumab was associated with manageable toxicities, with the most common grade 3-4 AEs being neutropenia, asthenia and nausea/vomiting. The incidence of grade 3-4 neutropenia (45.7\%) was lower than that reported in the C-TASKFORCE study $(72 \%)^{[9]}$ and the Danish study $(67 \%)^{[10]}$, and was slightly lower than in previous Japanese real-world studies $(48.2-52.4 \%)^{[14-16]}$. Furthermore, no patients developed febrile neutropenia in our study, while the incidence of this event was $16 \%$ and $6 \%$, respectively, in C-TASKFORCE and the Danish study ${ }^{[9,10]}$, and $0-3.3 \%$ in the Japanese realworld studies ${ }^{[14-16]}$. The lower levels of haematological toxicity observed in our study may have been due to the relatively high proportion of patients who received prophylactic G-CSF therapy (14.3\%). Of note, several studies have reported that chemotherapy-induced neutropenia with TAS-102 (with or without bevacizumab) is associated with improved survival outcomes ${ }^{[22-24]}$, which highlights the importance of 
G-CSF prophylaxis to prevent or manage neutropenia and allow for continued TAS-102 plus bevacizumab treatment without the need for dose reduction.

The limitations of our study include its retrospective, single-arm, single-centre design and its small population size ( $\mathrm{N}=35)$. An ongoing international phase III study (SUNLIGHT; NCT04737187) is currently investigating the efficacy and safety of TAS-102 plus bevacizumab versus TAS-102 monotherapy as third-line treatment in patients with refractory $\mathrm{mCRC}$, and has a target enrolment of 490 patients ${ }^{[25]}$. This open-label, multicentre study aims to further confirm the clinical benefits of TAS-102 plus bevacizumab over TAS-102 monotherapy in a large population of patients with refractory $\mathrm{MCRC}$; results are expected in 2023.

In conclusion, this real-world study confirms the efficacy and safety of TAS-102 plus bevacizumab in patients with refractory $\mathrm{mCRC}$ in routine clinical practice, with survival and tolerability outcomes that were generally consistent with previous clinical and real-world studies of patients in this setting.

\section{Methods}

\section{Study design}

This observational, retrospective, single-centre study was conducted at the A Coruña University Hospital in Spain in patients aged $>18$ years with a confirmed diagnosis of mCRC who were refractory or intolerant to standard therapies. Previous treatment included fluoropyrimidine, oxaliplatin and irinotecanbased chemotherapy and anti-EGFR agents (in patients with wild-type RAS/BRAF tumours). Eligible patients had received treatment with TAS-102 plus bevacizumab in routine clinical practice between July 2019 and October 2021, including patients who had previously received treatment with antiangiogenic agents (i.e. bevacizumab and/or aflibercept). Patients who had previously received TAS-102 monotherapy or TAS-102 in combination with antiangiogenic agents other than bevacizumab were excluded.

The standard doses administered at A Coruña University Hospital were TAS- $10230-35 \mathrm{mg} / \mathrm{m}^{2}$ on days 1-5 and days 8-12 every 28 days plus bevacizumab $5 \mathrm{mg} / \mathrm{kg}$ every 14 days. Starting treatment with reduced doses of TAS-102 or administration of prophylactic G-CSF was at the discretion of the treating physician.

The study was approved by the local ethics committee and conducted in accordance with the Declaration of Helsinki. All patients gave their informed consent prior to study inclusion.

\section{Data collection}

Clinical pathological characteristics and treatment data were collected from eligible patients' medical records, including sex, age and ECOG PS. Disease characteristics included RAS and BRAF mutational status, mismatch repair protein expression, primary tumour location, histological grade, tumour 
presentation (synchronous or metachronous), the number of metastatic locations ( $<3$ or $\geq 3$ ), the interval from metastatic disease diagnosis to TAS-102 plus bevacizumab initiation ( $\geq 18$ or $<18$ months), and the Tabernero prognostic classification (best, good or poor) ${ }^{[7]}$, as well as treatment history, including primary tumour resection, metastatic disease resection and previous treatments received. The start date and initial doses of TAS-102 plus bevacizumab, the use of prophylactic G-CSF, the number of cycles received, the response obtained (assessed by Response Evaluation Criteria in Solid Tumors version 1.1 criteria), disease progression and/or survival, toxicities according to Common Terminology Criteria for Adverse Events version 4.0, and any dose delays and/or dose reductions were also collected retrospectively from patient records for analysis.

\section{Study assessments}

OS was defined as the time between treatment initiation and death from any cause. PFS was defined as the interval between treatment initiation and radiological confirmation of disease progression or death from any cause. The ORR was defined as the proportion of patients who achieved CR or PR; the DCR was defined as the proportion of patients who achieved CR, PR, or stable disease for $\geq 6$ weeks after treatment initiation.

\section{Statistical analyses}

Statistical analyses were performed using SPSS statistics software version 25.0. The Chi-squared or Fisher's exact test (depending on the sample size) was used to compare patient clinical and demographic variables. The Kaplan-Meier model was used to estimate median PFS and OS and their $95 \% \mathrm{Cls}$. An analysis of potential predictors of PFS or OS was also conducted by comparing the differences between survival curves using univariate logistic regression and the log-rank test with a two-sided significance of < 0.05 .

\section{Declarations}

\section{Data Availability}

All data are available upon request from the corresponding author.

\section{Acknowledgments}

We would like to thank Sarah Greig, PhD, of Springer Healthcare Communications who provided editorial assistance in preparing the first draft of the manuscript. This medical writing assistance was funded by Fundación Profesor Novoa Santos.

\section{Author contributions}


N.M.L. contributed to study design, data analysis and drafting of the manuscript. All authors (N.M.L., T.C.C., B.A.D.C, R.V.P., C.R.R., M.I.G.-R.R., S.S.D, B.G.S., J. de la C.G., F.B.L., M.M.S. and M.R.L.) enrolled patients, collected clinical information, evaluated study data and critically reviewed the manuscript.

\section{Funding}

Biomedical Research Institute of A Coruña.

\section{Institutional Review Board Statement:}

Approved by Clinical Research Ethic Committee (CEIC) of Galicia, Spain.

\section{Competing interests}

The authors declare no competing interests.

\section{Additional information}

Correspondence and requests for materials should be addressed to N.M.L.

\section{References}

1. Bray, F. et al. Global cancer statistics 2018: GLOBOCAN estimates of incidence and mortality worldwide for 36 cancers in 185 countries. CA Cancer J Clin 68, 394-424, https://doi.org/10.3322/caac.21492 (2018).

2. Van Cutsem, E. et al. ESMO consensus guidelines for the management of patients with metastatic colorectal cancer. Ann Onco/ 27, 1386-1422, https://doi.org/10.1093/annonc/mdw235 (2016).

3. Carlomagno, C. et al. Multiple treatment lines and prognosis in metastatic colorectal cancer patients. Cancer Metastasis Rev 38, 307-313, https://doi.org/10.1007/s10555-018-9748-7 (2019).

4. Mayer, R. J. et al. Randomized trial of TAS-102 for refractory metastatic colorectal cancer. $N$ Engl J Med 372, 1909-1919, https://doi.org/10.1056/NEJMoa1414325 (2015).

5. Xu, J. et al. Results of a randomized, double-blind, placebo-controlled, phase III trial of trifluridine/tipiracil (TAS-102) monotherapy in Asian patients with previously treated metastatic colorectal cancer: the TERRA study. J Clin Onco/ 36, 350-358, https://doi.org/10.1200/JC0.2017.74.3245 (2018).

6. Yoshino, T. et al. TAS-102 monotherapy for pretreated metastatic colorectal cancer: a doubleblind, randomised, placebo-controlled phase 2 trial. Lancet Oncol 13, 993-1001, 
https://doi.org/10.1016/S1470-2045(12)70345-5 (2012).

7. Tabernero, J. et al. Effect of trifluridine/tipiracil in patients treated in RECOURSE by prognostic factors at baseline: an exploratory analysis. ESMO Open 5, e000752, https://doi.org/10.1136/esmoopen2020-000752 (2020).

8. Ishizaki, T. et al. Prospective multicenter phase II study of biweekly TAS-102 and bevacizumab for metastatic colorectal cancer. Anticancer Res 41, 2157-2163, https://doi.org/10.21873/anticanres.14988 (2021).

9. Kuboki, Y. et al. TAS-102 plus bevacizumab for patients with metastatic colorectal cancer refractory to standard therapies (C-TASK FORCE): an investigator-initiated, open-label, single-arm, multicentre, phase 1/2 study. Lancet Onco/ 18, 1172-1181, https://doi.org/10.1016/S14702045(17)30425-4 (2017).

10. Pfeiffer, P. et al. TAS-102 with or without bevacizumab in patients with chemorefractory metastatic colorectal cancer: an investigator-initiated, open-label, randomised, phase 2 trial. Lancet Oncol 21, 412-420, https://doi.org/10.1016/S1470-2045(19)30827-7 (2020).

11. Satake, H. et al. Phase Ib/ll study of biweekly TAS-102 in combination with bevacizumab for patients with metastatic colorectal cancer refractory to standard therapies (BiTS Study). Oncologist 25, e1855-e1863, https://doi.org/10.1634/theoncologist.2020-0643 (2020).

12. Takahashi, T. et al. Phase II study of trifluridine/tipiracil plus bevacizumab by RAS mutation status in patients with metastatic colorectal cancer refractory to standard therapies: JFMC51-1702-C7. ESMO Open 6, 100093, https://doi.org/10.1016/j.esmoop.2021.100093 (2021).

13. Yoshida, Y. et al. Combination of TAS-102 and bevacizumab as third-line treatment for metastatic colorectal cancer: TAS-CC3 study. Int J Clin Onco/ 26, 111-117, https://doi.org/10.1007/s10147-020-01794-8 (2021).

14. Chida, K. et al. Efficacy and safety of trifluridine/tipiracil plus bevacizumab and trifluridine/tipiracil or regorafenib monotherapy for chemorefractory metastatic colorectal cancer: a retrospective study. Ther Adv Med Oncol 13, 17588359211009143, https://doi.org/10.1177/17588359211009143 (2021).

15. Fujii, $\mathrm{H}$. et al. Bevacizumab in combination with TAS-102 improves clinical outcomes in patients with refractory metastatic colorectal cancer: a retrospective study. Oncologist 25, e469-e476, https://doi.org/10.1634/theoncologist.2019-0541 (2020).

16. Kotani, D. et al. Retrospective cohort study of trifluridine/tipiracil (TAS-102) plus bevacizumab versus trifluridine/tipiracil monotherapy for metastatic colorectal cancer. BMC Cancer 19, 1253, https://doi.org/10.1186/s12885-019-6475-6 (2019). 
17. Fernández Montes, A. et al. Prediction of survival in patients with advanced, refractory colorectal cancer in treatment with trifluridine/tipiracil: real-world vs clinical trial data. Sci Rep 11,14321, https://doi.org/10.1038/s41598-021-93732-5 (2021).

18. Yang, L. et al. Comparisons of metastatic patterns of colorectal cancer among patients by age group: a population-based study. Aging 10, 4107-4119, https://doi.org/10.18632/aging.101700 (2018).

19. Wang, J. et al. Metastatic patterns and survival outcomes in patients with stage IV colon cancer: A population-based analysis. Cancer Med 9, 361-373, https://doi.org/10.1002/cam4.2673 (2020).

20. Matsuhashi, N. et al. Combination chemotherapy with TAS-102 plus bevacizumab in salvage-line treatment of metastatic colorectal cancer: a single-center, retrospective study examining the prognostic value of the modified Glasgow Prognostic Score in salvage-line therapy of metastatic colorectal cancer. Mol Clin Oncol 11, 390-396, https://doi.org/10.3892/mco.2019.1899 (2019).

21. Kuramochi, H. et al. The pre-treatment lymphocyte-to-monocyte ratio predicts efficacy in metastatic colorectal cancer treated with TAS-102 and bevacizumab. Anticancer Res 41, 3131-3137, https://doi.org/10.21873/anticanres.15098 (2021).

22. Kasi, P. M. et al. Chemotherapy induced neutropenia at 1-month mark is a predictor of overall survival in patients receiving TAS-102 for refractory metastatic colorectal cancer: a cohort study. BMC Cancer 16, 467, https://doi.org/10.1186/s12885-016-2491-y (2016).

23. Nose, Y. et al. Neutropenia is an indicator of outcomes in metastatic colorectal cancer patients treated with FTD/TPI plus bevacizumab: a retrospective study. Cancer Chemother Pharmaco/ 86, 427433, https://doi.org/10.1007/s00280-020-04129-6 (2020).

24. Yoshino, T. et al. Neutropenia and survival outcomes in metastatic colorectal cancer patients treated with trifluridine/tipiracil in the RECOURSE and J003 trials. Ann Onco/ 31, 88-95, https://doi.org/10.1016/j.annonc.2019.10.005 (2020).

25. Tabernero, J. et al. Trifluridine/tipiracil plus bevacizumab for third-line management of metastatic colorectal cancer: SUNLIGHT study design. Future Oncol 17, 1977-1985, https://doi.org/10.2217/fon-2020-1238 (2021).

\section{Figures}

\section{Figure 1}

Kaplan-Meier curves for (a) progression-free survival (PFS) and (b) overall survival (OS). 